\title{
Design and Implementation of AES Data Security Protocol
}

\author{
Dinesh B. Bhoyar ${ }^{1}$, Indersheel Kaur'2, Swati K. Mohod ${ }^{3}$ and K. D. Kulat ${ }^{4}$ \\ ${ }^{1}$ Assistant Professor, Department of ETC, Yeshwantrao Chavan \\ College of Engineering, Nagpur, Maharashtra, India \\ ${ }^{2} P G$ Scholar, Department of ETC, Yeshwantrao Chavan \\ College of Engineering, Nagpur, Maharashtra, India \\ ${ }^{3}$ Assistant Professor, Department of EL, Yeshwantrao Chavan \\ College of Engineering, Nagpur, Maharashtra, India \\ ${ }^{4}$ Professor, Department of ECE, Visvesvaraya National \\ Institute of Technology, Nagpur, Maharashtra, India
}

\section{ABSTRACT}

Data Security is in top priority when we transmit the data through internet. In this paper AES (Advanced Encryption Standard) algorithm is implemented using FPGA for security of IoT data. We used symmetric key block cipher for data encryption and decryption. The power consumption and area required for FPGA implementation is less. The Xilinx FPGA device Xc7v285tl-1L ffg 1157is used for implementation

KEY WORDS: AES, FPGA, IOT, ENCRYPTION, DECRYPTION, XILINX.

\section{INTRODUCTION}

Security is one of the most important concerns of data transmission through Internet. The internet security protocols take care of the issues related to data security. In IoT [1] through its own architecture and devices it gives the solution to the data secutity. The data security solution has direct relation to the cost and time to market. However, every solution has its own strength and weaknesses. Thus every IoT solution does not require the entire IoT security protocols. The main focus of this paper is to implement the AES [2][3] algorithm using FPGA for security of IoT data. The paper is divided into four different section, the first section gives the introduction

\section{ARTICLE INFORMATION}

*Corresponding Author: dinesh.bhoyar23@gmail.com Received 20th Oct 2020 Accepted after revision 29th Dec 2020 Print ISSN: 0974-6455 Online ISSN: 2321-4007 CODEN: BBRCBA

Thomson Reuters ISI Web of Science Clarivate Analytics USA and Crossref Indexed Journal

\section{1) Clarivate $\begin{gathered}\text { Analytics } \\ \text { rossef }\end{gathered}$}

NAAS Journal Score 2020 (4.31)

A Society of Science and Nature Publication,

Bhopal India 2020. All rights reserved.

Online Contents Available at: http//www.bbrc.in/

Doi: $h$ ttp://dx.doi.org/10.21786/bbrc/13.14/111 whereas second, third and fourth section focuses on the hardware implementation, result and conclusion.

2. Hardware implementation: The Encryption and Decryption process in each round is as per the fig-1.

a. AddSubbytes: Each entry in the $\mathrm{S}$ box is replaced by the add sub byte. Non linearity in the cipher is also provided by add sub byte. To derive the S-box multiplicative inverse iGF (28) is used. The combination of inverse function with an invertible transformation gives S-box. While designing the decryption process the inverse of subbytes is used.

b. Shiftrows: In this, the bytes in rows shifted left cyclically. Every row is shifted with respect to the subsequent rows. In AES algorithm the shifting operation will be performed from the second row. The rows are shifted as follows:

2nd row is shifted by 1

3rd row is shifted by 2

4 th row is shifted by 3 and so on

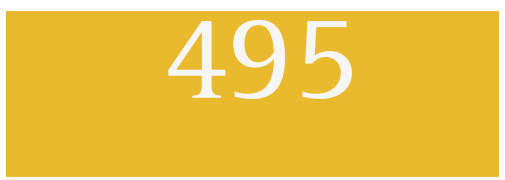


Figure 1: Flow Chart of Encryption and Decryption Process

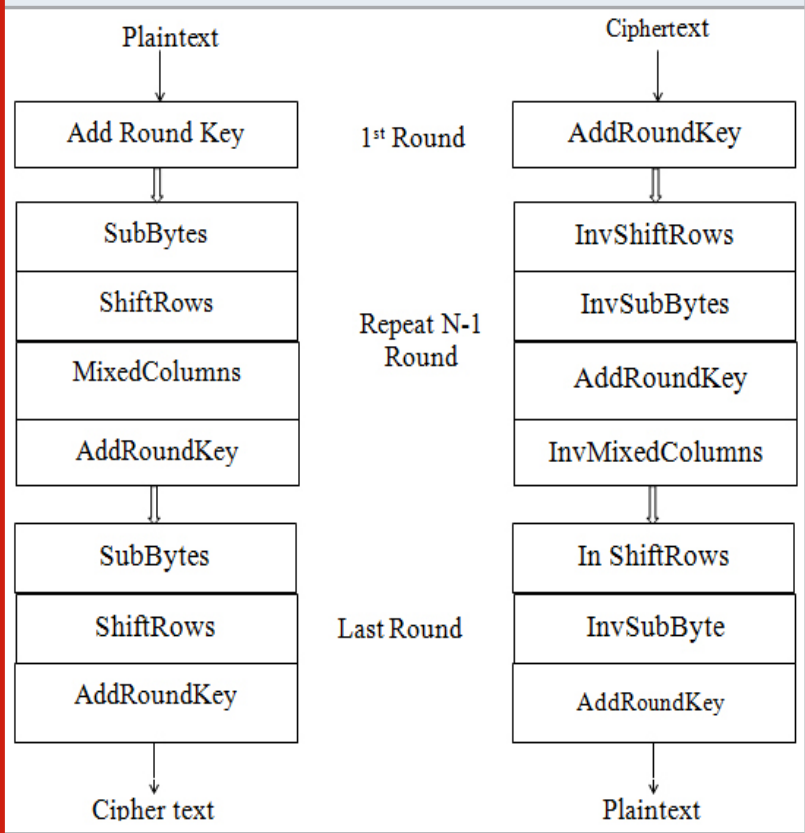

Figure 2: Proposed FPGA Implementation Module [6]

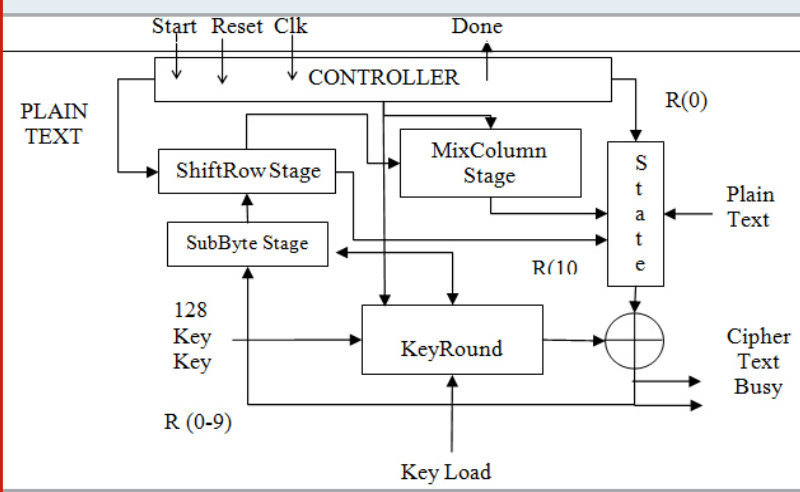

c. Mixcolumns: Each column is multiplied by the fixed polynomial. By using an invertible linear transformation the four bytes of each column of the state are combined.

d. Addround key: The XOR operation [4] will be performed between each and every state and 8 bit data of round sub key. The output is combined with the subkey. The subkey is added with each byte of the state. It is done by using XORing operation. A required subkey is derived from the main key.

e. Keyexpansion: The key expansions are based on ROTWORD, SUBWORD, RCON and XOR The key expansion algorithm is used to generate linear array of 44 words i.e 176 bytes. The input to the algorithm is a four word (16 bytes) for generating this. It provides four-word round key which is used for initial operation of AddRoundKey and each of the 10 rounds of the cipher. The key is copied into the first four words of the expanded key to derive the S-box. [5]. The S-Box is the output of the combination of inverse function and invertible transformation. The Decryption operation is exactly reverse operation of Encryptions

\section{RESULTS}

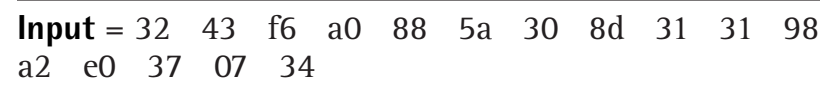

Cipher Key = 2b 7 e $15 \quad 16 \quad 28$ ae $\quad$ d2 $\quad$ a6 ab f7 15 8809 cf $4 f \quad 3 c$

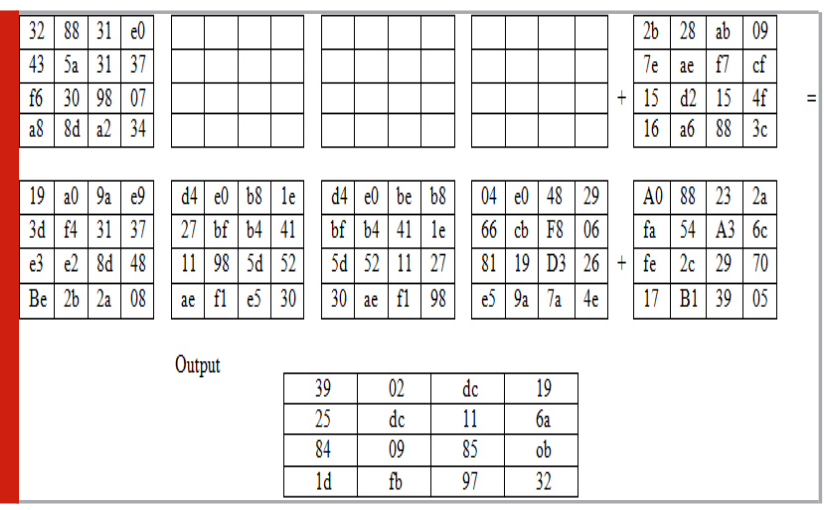

\section{a. Result after subbytes and shift rows}

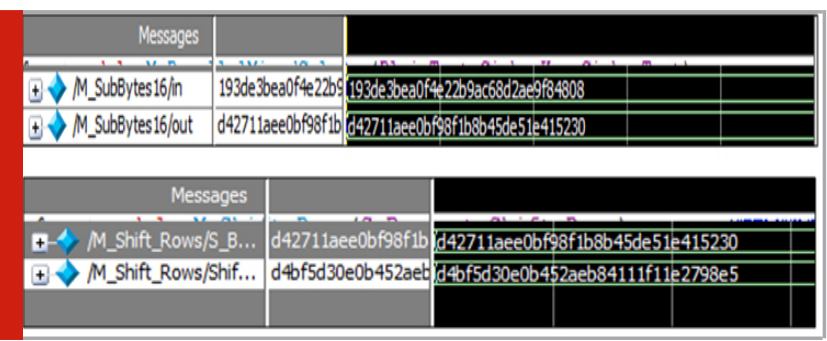

\section{b. Results after mix columns}

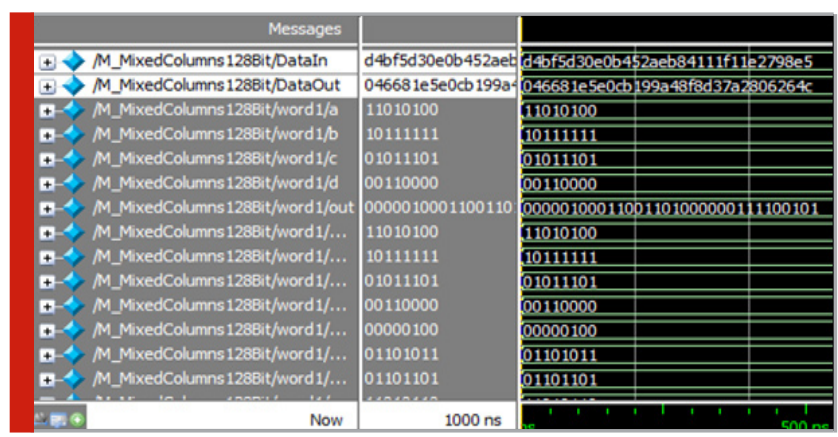

\section{c. Result after adding round key}

\section{Messages}

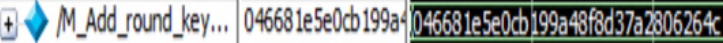

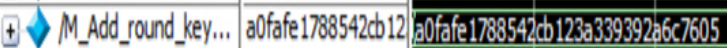

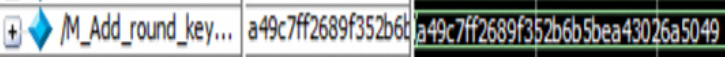




\section{c. Results after encryption with binary input}

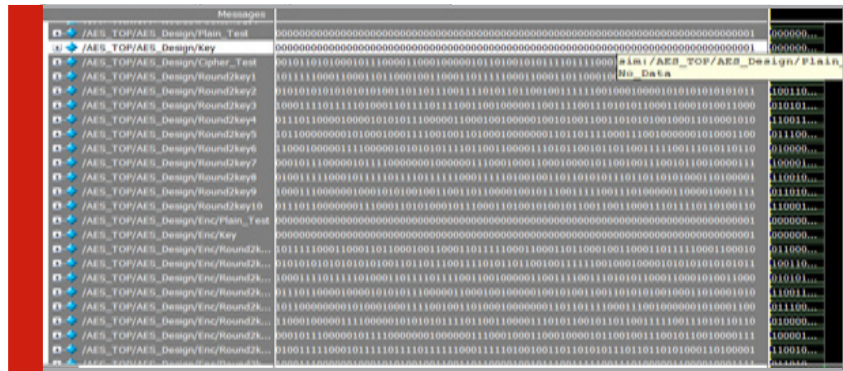

\section{d. Result After Encryption with hexadecimal input}

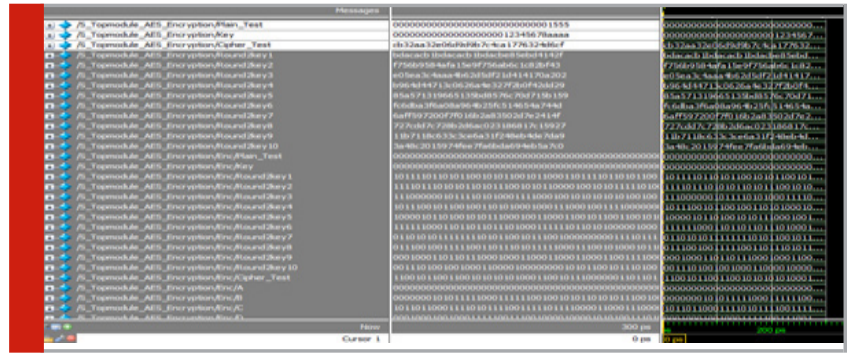

\section{e. Result after Decryption}

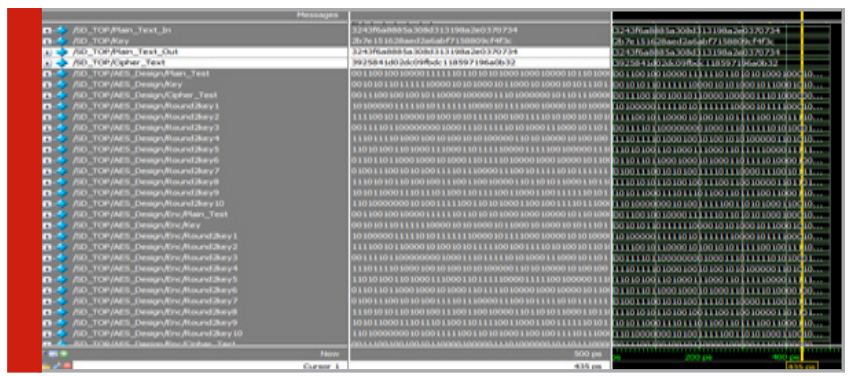

Testing and Verification: The following Table-1 [8] gives the device utilization summary of number of CLBs, IOBs and LUTs used for implementation of the proposed algorithm of AES-128.

\section{CONCLUSION}

The AES is also called as block cipher algorithm. For encryption and decryption 128-bit key is used. The AES code is designed and developed using VERILOG HDL. The final design is verified using XILINX ISE tool. This FPGA implemented design takes less area, less power and operated at a high speed. The developed design

\begin{tabular}{l|c|c|c|} 
Table 1 \\
\hline \multirow{2}{*}{$\begin{array}{l}\text { FPGA Device } \\
\text { Allocated Area }\end{array}$} & \multicolumn{3}{|c|}{ Xc7v285tl-1L ffg 1157} \\
\cline { 2 - 4 } & Available & Used & $\%$ of utilization \\
\hline CLB Slices & 357,600 & 2900 & 0.8 \\
\hline No. of LUT & 178,800 & 5702 & 3.1 \\
\hline IOBs & 600 & 385 & 64 \\
\hline
\end{tabular}

can be implemented in ASIC and used in low battery operated devices such as Bluetooth and wireless operated devices.

\section{REFERENCES}

AmitKumar, ManojKumar,P. Balramudu."Implementation of AES algorithm using VHDL”, 2017 International Conference on Computing Methodologies and Communication

Abdelali El Bouchti, Samir Bahsani, Trik Nahhal "Encryption as a Service for Data Healthcare Cloud Security “, Fifth International Conference on Future Generation Communication Technologies

Dinesh B. Bhoyar, Shelly R. Wankhede, Swati K. Modod. "Chapter 40 Design and Implementation of AES on FPGA for Security of IOT Data”, Springer Science and Business Media LLC, 2020

Dinesh Bhoyar, Barkha Katey, Manish Ingale “ LoRa technology based low cost water meter reading system"Esvier SSRn Information Systems and Ebusiness Neyworks ISSN 1556-5066

L.Thulasimani and M.Madheswaran," A single chip design and implementation of AES -128/192/256 encryption algorithms" International Journal of Engineering Science and Technology Vol. 2(5), 2010, 1052-1059

R. L. Rivest, A. Shamir and L. Adleman, "A method for obtaining digital signatures and public- key cryptosystem,'• Common. ACM, vol.21 no. 2, pp. 120126.

Y Chandu, K. S. Rakesh Kumar, Ninad Vivek Prabhukhanolkar, A N Anish, Sushma Rawal "Design and implementation of hybrid encryption for security of IOT data”, 2017 International Conference On Smart Technologies for Smart Nation 2017 